\title{
A General Approach to the Statistical Mechanics of Disordered Binary Alloys- Theory and Applications
}

\author{
By S.W. WILKINS \\ Department of Physics, Imperial College, $\equiv$ lon SW7, England \\ AND C. G. SHIRLEY \\ Department of Metallurgyand Materials Science, Carnegie-Mellon University, Pittsburgh,Pennsylvania 15213, U.S.A.
}

(Received 22 May 1974; accepted 21 September 1974)

\begin{abstract}
Some features and results of a general approach to the statistical mechanics of disordered binary alloys are briefly outlined. The general approach assists the authors to formulate criteria for the validity of the generalized mean-field model used for determining pair interaction energies from diffuse-scattering data. Some values for these pair interaction energies in certain alloys are presented and discussed.
\end{abstract}

\section{Introduction}

One of the most fundamental types of information which one can hope to acquire about alloys is the nature of the interparticle interaction energies. Statistical-mechanical theories of ordering, valid for substitutional binary alloys in thermal equilibrium above the critical temperature $T_{c}$, provide a means for deter- mining the pairwise interaction energies in an alloy from experimentally measured short-range-order parameters or site-occupation pair-correlation functions (see for example Cowley \& Wilkins, 1972; Moss \& Clapp, 1968; Wilkins, 1970; Wu \& Tahir-Kheli, 1971). The present paper first gives a brief outline of a general approach to the statistical mechanics of disordered alloys (to be described more fully in a forthcoming 
paper), with the aim of providing a framework within which it is possible to critically evaluate the accuracy of various ordering theories.

Of particular importance from the viewpoint of determining pairwise interactions is the result that the present method enables one to list criteria (see $\S$ II) for selecting alloys and experimental conditions such that the generalized mean-field expression used for the Fourier inverse of the pair-correlation functions (defined in next section)

$$
S(\mathbf{k})=G_{2}(T) /\left[1+G_{1}(T) V(\mathbf{k})\right]
$$

should be a good approximation. In (1) $V(\mathbf{k})$ is the Fourier inverse of the usual pairwise intersite interaction energies $V_{0}$ whilst $G_{1}(T)$ and $G_{2}(T)$ are essentially temperature-dependent functions.

Some results for the first seven pair-interaction ratios for certain alloys, which were derived via (1), are presented and discussed in $\S$ III. A comparison is made between these results for the pair interactions and the form of the long-range interaction attributed to the conduction-electron screening of the ions.

\section{Statistical mechanics}

The detailed formulation of the statistical-mechanical problem for equilibrium binary alloys has been given in Shirley \& Wilkins (1972) and will not be repeated here. It is, however, desirable for the present discussion of the development of approximate ordering theories to recall that the configurations of the alloy were described there using site occupation variables

$$
\sigma_{i}=\left\{\begin{array}{r}
2 m_{\mathrm{B}} \text { for an A atom at site } i \\
-2 m_{\mathrm{A}} \text { for a B atom at site } i,
\end{array}\right.
$$

which have many useful properties including the one that

$$
\langle\sigma\rangle_{c}=0
$$

over any possible configuration of the $\left(m_{\mathbf{A}}, m_{\mathbf{B}}\right)$ composition alloy. Also, it is assumed here that the usual pairwise Hamiltonian (see, e.g., Clapp \& Moss, 1966) is a sufficiently accurate model.

The quantities of interest are the statistical mechanical averages $\left\langle\sigma_{t} \sigma_{j}\right\rangle$ which in the grand canonical ensemble are defined as

$$
\left\langle\sigma_{i} \sigma_{J}\right\rangle=\sum_{\left\{\sigma_{f}\right\}} \sigma_{i} \sigma_{j} \varrho / \sum_{\left\{\sigma_{f}\right\}} \varrho,
$$

where $\varrho=\exp \left\{-\beta H+\lambda \sum_{\theta} \sigma_{g}\right\}$, with $\beta=1 / k_{B} T$ and $H$ the pairwise Hamiltonian. The summation is over all possible sets $\left\{\sigma_{f}\right\}$ where each $\sigma$ may independently take on the values $2 m_{\mathrm{B}}$ and $-2 m_{\mathrm{A}}$, subject only to the constraint that the chemical potential (Lagrange multiplier) $\lambda$ is eventually determined such that the composition constraint $\left\langle\sigma_{f}\right\rangle=0$ is satisfied, to be consistent with the known property (3).
By expanding correlation functions of type (4) one obtains the following doubly infinite hierarchy of coupled linear equations for the correlation functions (see Shirley \& Wilkins, 1972)

$$
\begin{aligned}
\left\langle\sigma_{0}\right\rangle \equiv\langle o\rangle=0 & =D_{0}(t) \\
& +\frac{1}{4} \beta^{2} D_{2}(t) \sum_{f g} V_{0 f} V_{0 g}\langle f g\rangle-\ldots \\
\left\langle\sigma_{0} \sigma_{i}\right\rangle \equiv\langle o i\rangle= & -\frac{1}{2} \beta D_{1}(t) \sum_{f} V_{0 f}\langle f i\rangle \\
& +\frac{1}{4} \beta^{2} D_{2}(t) \sum_{f g} V_{0 f} V_{0 g}\langle f g i j\rangle-\ldots \\
\left\langle\sigma_{0} \sigma_{i} \sigma_{j}\right\rangle \equiv\langle o i j\rangle & =D_{0}(t)\langle i j\rangle-\frac{1}{2} \beta D_{1}(t) \sum_{f} V_{0 f}\langle f i j\rangle \\
& +\frac{1}{4} \beta^{2} D_{2}(t) \sum_{f g} V_{0 f} V_{0 g}\langle f g i j\rangle-\ldots
\end{aligned}
$$

etc., where all $o, i, j, \ldots$ are distinct. The expansion coefficients in (5) are given by

$$
\begin{gathered}
D_{0}(t)=t-\left(m_{\mathrm{A}}-m_{\mathrm{B}}\right) \\
D_{m}(t)=\frac{\mathrm{d}^{m} \tanh (\lambda)}{\mathrm{d} \lambda^{m}}, \quad(\text { for } m>0)
\end{gathered}
$$

with

$$
t \equiv \tanh (\lambda)
$$

and $\lambda$ (or $t$ ) must be chosen to satisfy $\langle\sigma\rangle=0$. The derivation of equations (5) has not involved any approximations of a mathematical kind.

In order to make some progress with the approximate solution of the statistical-mechanical problem, it is assumed for the present that all pair interactions favour clustering (i.e. $V_{0 i} \leq 0$ for all $i$ ). This assumption enables the use of the order-in-1/z classification scheme developed by Shirley \& Wilkins (1972) to help truncate (5) correct to arbitrary order in $1 / z$, where $z$ is the number of sites interacting with a given site.

It is found that (5) is an implicit expansion in $1 / z$ both horizontally from left to right, and vertically from above to below. For example, if $(5 b)$ is truncated at the first term the simple $\mathrm{O}(1 / z)$ (Clapp \& Moss or mean-field) approximation is obtained, and involves pair correlations alone. However, if equations (5) are truncated correctly to higher orders in $1 / z$, one will in general obtain a coupled set of linear equations involving irreducible (all sites distinct) many-site correlation functions.

It is thus useful to find (see Wilkins, 1972; Wilkins \& Shirley, 1974) that one may decouple irreducible many-site correlation functions correctly to leading order in $1 / z$ as follows:

(i) for irreducible $2 n$-site correlation functions

$$
\begin{aligned}
\langle 123 \ldots(2 n-1)(2 n)\rangle & =\{\langle 12\rangle\langle 34\rangle \ldots\langle(2 n-1)(2 n)\rangle\} \\
\text { e.g. } \quad & \\
\langle 1234\rangle=\langle 12\rangle & \langle 34\rangle+\langle 13\rangle\langle 24\rangle+\langle 14\rangle\langle 23\rangle ;
\end{aligned}
$$


(ii) for irreducible $(2 n+1)$-site correlation functions

$$
\begin{aligned}
\langle 123 \ldots(2 n) & (2 n+1)\rangle \\
= & \{\langle 123\rangle\langle 45\rangle \ldots\langle(2 n)(2 n+1)\rangle\},
\end{aligned}
$$

where the brace denotes the sum over all distinct permutations of the site labels. The proof of (8) (Wilkins $\&$ Shirley, 1974) involves the use of the order-in- $1 / z$ classification scheme and some algebraic manipulation of equation (5).

In the case of equiatomic $\left(m_{A}=\frac{1}{2}\right)$ alloys it is possible (Wilkins \& Shirley, 1974) to use the decoupling scheme $(8 a)$ to truncate equation $(5 b)$ correct to $\mathrm{O}\left(1 / z^{2}\right)$ and also to sum a whole class of higher-order terms [note that only terms involving $2 n$-site correlation functions in $(5 b)$ are non-vanishing for $m_{A}=\frac{1}{2}$ ]. Moreover, it is found there that this approximation for $\langle o i\rangle$ is of the form (1) and incorporates the spherical-model result [thus constituting an alternative derivation of a result obtained earlier by Brout (1961)]. Also for the equiatomic case, it is possible to find a more accurate decoupling scheme for irreducible four-site correlations than is given by $(8 a)$. The use of this improved result to decouple $(5 b)$ is found to lead to an ordering theory which departs in $\mathrm{O}\left(1 / z^{3}\right)$ from the simple functional form (1) which incorporated both the $\mathrm{O}(1 / z)$ and $\mathrm{O}\left(1 / z^{2}\right)$ results (Wilkins \& Shirley, 1974).

In the non-equiatomic case the simple functional form (1) is found to break down even in $\mathrm{O}\left(1 / z^{2}\right)$, but only weakly for $m_{\mathrm{A}} \simeq \frac{1}{2}$.

Detailed investigation of equations (5), including that described above, leads to the conclusion that the basic

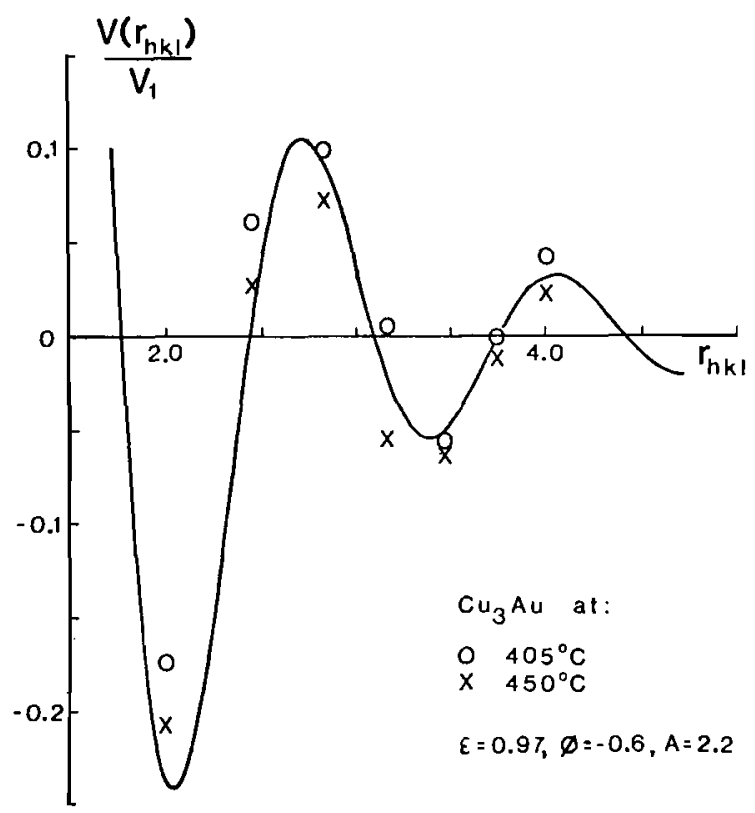

Fig. 1. Values for the pair-interaction ratios $V_{l} / V_{1}$ deduced from the short-range-order parameters of Moss (1964) for $\mathrm{Cu}_{3} \mathrm{Au}$ at two temperatures, compared with the free-electron screening curve, equation (9), for the indicated parameters. functional form (1) should be most reliable as a mathematical approximation to the statistical-mechanical problem with pairwise Hamiltonian for:

(i) $T$ not too close to $T_{c}$ (several percent away at least;

(ii) $m_{\mathrm{A}} \simeq \frac{1}{2}$, i.e. concentrated alloys;

(iii) $V_{i} \leq 0$ for all $i$, i.e. completely (or at least strongly) clustering alloys or for $V_{1}>0$ when it is the dominant interaction and especially when the lattice is of the loose packed variety (e.g. simple cubic or b.c.c., but not f.c.c.);

(iv) large $z$, i.e. long-range (completely clustering) interactions.

It is unfortunate to have to note from the above that the conditions under which the generalized functional form (1) for $S(\mathbf{k})$ should be best are, in general, those for which the separation procedure for extracting the reduced short-range-order diffuse scattering $S_{\text {expt }}(\mathbf{k})$ from the total measured diffuse scattering is least reliable. This provides a motivation for the extension of the statistical-mechanical theory of binary alloys to a wider domain of validity so as rigorously to include both dilute and non-clustering alloys, and also to treat more general Hamiltonians than the usual pairwise one (see, e.g., Shirley, 1974).

\section{Determination of pair-interaction ratios}

Equation (1) provides a means whereby experimental data on the short-range-order diffuse scattering may be reverted to obtain information about the pair interactions in a given alloy. In practice it is simpler to do the analysis in direct space and the details of the method are sufficiently described elsewhere (Cowley \& Wilkins, 1972; Wilkins, 1970). The purpose of the present section is partly to review the results which have already been obtained and also to compare these with some new results for $\mathrm{CuAu}$ obtained from the data of Metcalfe (1973), which could provide evidence for the inadequacy of doing the analysis with equation (1).

The pair interaction for the $i$ th neighbour shell is denoted by $V\left(\mathbf{R}_{0 i}\right)=V_{i}$. From the published data for various alloys, results for the first seven pair-interaction ratios $\left(V_{2} / V_{1}, V_{3} / V_{1}, \ldots\right)$ have been determined for:-

(i) $\mathrm{Cu}_{3} \mathrm{Au}$ at 405 and $450^{\circ} \mathrm{C}$ from the first eleven short-range-order parameters obtained by Moss (1964). The results for this system are reproduced in Fig. 1.

(ii) $\mathrm{CuAu}$ at $800^{\circ} \mathrm{K}$ from only the first fourteen short-range order parameters obtained by Metcalfe (1973). The results for this system are presented in Fig. 2.

(iii) $\mathrm{Au}-40$ at. \% Pd. at room temperature from the data of Lin, Sprueill \& Williams (1970). The results for this system are presented in Fig. 3.

The method of deriving the pair-interaction ratios is independent of any assumed model for their origin. Thus an unprejudiced comparison can be made with the free-electron screening model (e.g. see Blandin \& 
Deplanté, 1963) which gives an effective pair-interaction energy $V\left(\mathbf{R}_{o i}\right)$ between ions at large distances as

$$
V\left(r_{h k l}\right) / V_{1}=A \cos \left[2 k_{F} a_{0} r_{h k l}+\phi\right] / r_{h k l}^{3},
$$

where $k_{F}$ is the Fermi wave number, $A$ is assumed to be a constant, and $\phi$ is a phase factor. The unit-cell dimension is $a_{0}$ and $r_{k k l}$ is the interatomic distance in units of half the unit-cell axes for lattice sites with integral coordinates $h, k, l$. Comparison with the more exact result for a small impurity potential suggests that at metallic electron densities this form should be good beyond the first nearest-neighbour distance. The Fermi wave number is proportional to $\varepsilon$, which is the cube root of the average number of conduction electrons per atom.

Comparison with the results for $\mathrm{Cu}_{3} \mathrm{Au}$ in Fig. 1 shows that the curve for $\varepsilon=0.97$ and $\phi=-0.6$ appears to fit the experimental data very well. Also, the results for the $V_{i}$ ratios have been found to be quite stable to variation of the weighting factors in the least-squares analysis.

For $\mathrm{CuAu}$ the results in Fig. 2 are compared with the screening curve for $\varepsilon=1 \cdot 0$ and $\phi=-0 \cdot 79$. Although these parameter values are almost the same as for $\mathrm{Cu}_{3} \mathrm{Au}$, the agreement is not nearly so good, especially beyond fourth-neighbour interactions. Also, the results have not been found to be as stable to variation in weighting factor as those for $\mathrm{Cu}_{3} \mathrm{Au}$.

For $\mathrm{Au}-40$ at. \% $\mathrm{Pd}$ the results in Fig. 3 are compared with the screening curve for $\varepsilon=0.844$ and $\phi=0.5$. The best-fit value of $\varepsilon$ adopted there is close to the value $\varepsilon=0.825$ obtained from a theoretical $d$-bandfilling argument where each $\mathrm{Pd}$ atom contributes 0.55 positive holes to the $d$ band per Pd atom (Mott \& Jones, 1936, p. 199). Agreement for this case also is far from perfect. Moreover, the results have not been found to be as stable to variation of the weighting factors as those for $\mathrm{Cu}_{3} \mathrm{Au}$. The value of $\varepsilon$ used for Fig. 3 is different from that used in Cowley \& Wilkins (1972) the good agreement between the value adopted there and the Kohn-anomaly argument no longer holds.

The crucial question which one must ask about $\mathrm{CuAu}$ and the Au-Pd alloy is whether these differences between the theoretical screening curve and the values derived from experiment are: (i) real differences, (ii) errors in the data and data refinement procedure or (iii) limitations of the theory, and in particular the assumption of the pairwise model (4) and the functional form (1).

In the case of $\mathrm{CuAu}$, the difference between the interaction ratios for this system and those for $\mathrm{Cu}_{3} \mathrm{Au}$ is more than can readily be ascribed to a simple lattice-parameter change (Moss \& Clapp, 1968; Wu \& Tahir-Kheli, 1971). This would appear to leave the possibilities of errors in the data and limitations of the theory wide open. Since $\mathrm{CuAu}$ is an equiatomic alloy it is possible to use the more accurate mathematical result discussed earlier to test whether the interaction ratios thus determined are significantly different from those determined with equation (1). However, $\mathrm{CuAu}$ appears somewhat anomalous in as much as $V_{2}$ and $V_{4}$ are almost equal in magnitude to $V_{1}$, thus condition (iii) in the criteria for the validity of (1) is severely tested. Ideally one would like to test the more accurate theory on order parameters measured for an equiatomic clustering alloy such as $\mathrm{CuNi}$ or $\mathrm{AuNi}$.

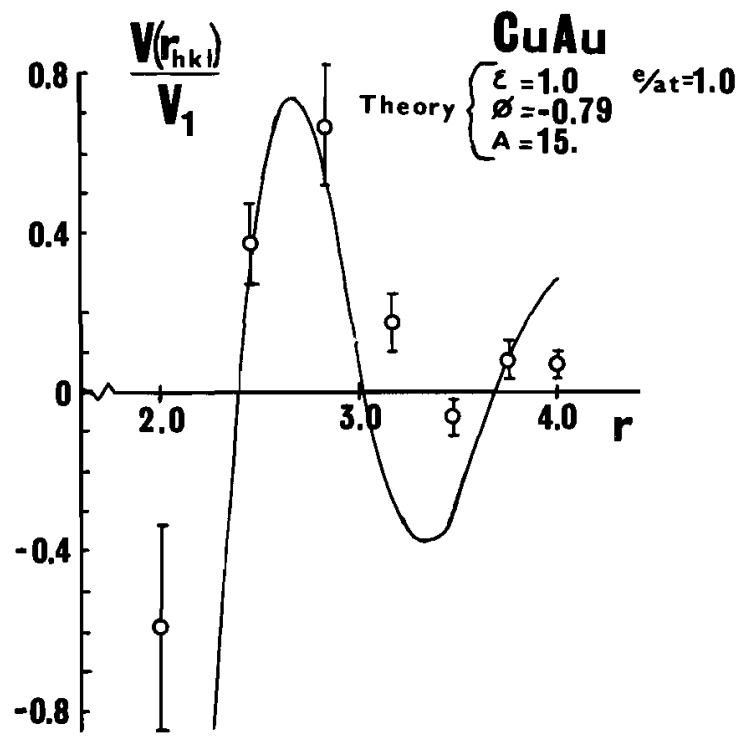

Fig. 2. Values for the pair-interaction $V_{t} / V_{1}$ deduced from the short-range-order parameters of Metcalfe (1973) for $\mathrm{CuAu}$ at $800^{\circ} \mathrm{K}$, compared with the free-electron screening curve, equation (9), for the indicated parameters.

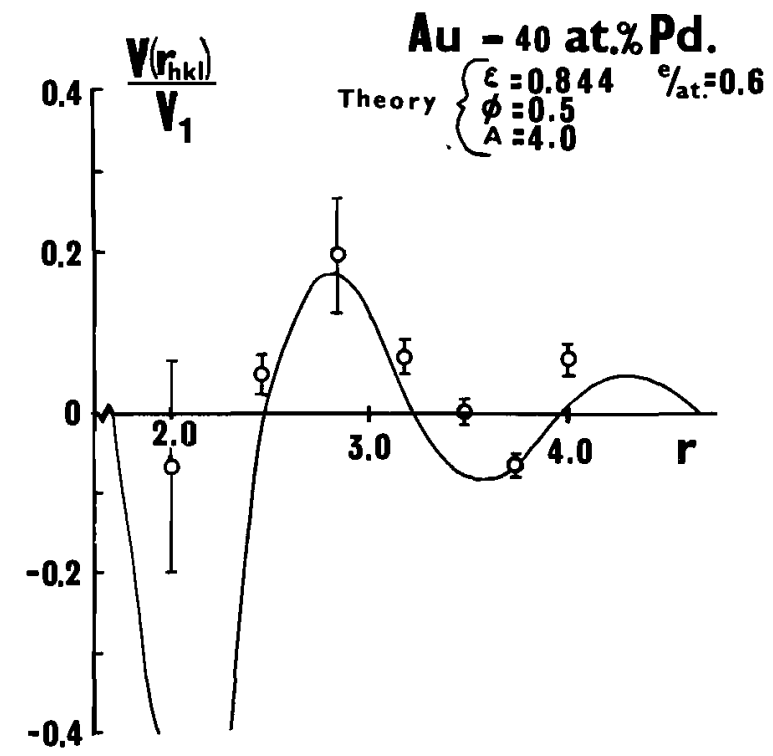

Fig. 3. Values for the pair-interaction ratios $V_{i} / V_{1}$ deduced from the short-range-order parameters of Lin, Sprueill \& Williams (1970) for $\mathrm{Au}-40$ at. \% Pd at room temperature, compared with the free-electron screening curve, equation (9), for the indicated parameters. 
It is only by more detailed experimental and numerical investigation that the question of where, if anywhere, the errors lie can be answered conclusively. However, by knowing the criteria for greatest validity of both thenry and experiment one will be more readily able to make this decision. It is to the former consideration that this paper has principally been directed.

The authors are grateful to Dr Tibballs for pointing out the $d$-band-filling argument for $\mathrm{Au}-\mathrm{Pd}$ alloys and also to $\mathrm{Dr}$ Metcalfe for permission to use his $\mathrm{CuAu}$ data prior to publication and for valuable discussions. One of us (SWW) wishes to gratefully acknowledge financial support from the Sir Arthur Sims Travelling Scholarship and a grant from the Science Research Council.

\section{References}

Blandin, A. \& Deplanté, J. L. (1963). In Metallic Solid Solutions, edited by J. Friedel and A. Guinier. New York: Benjamin.
Brout, R. (1961). Phys. Rev. 122, 469-474.

Clapp, P. C. \& Moss, S. C. (1966). Phys. Rev. 142, 418427.

CoWley, J. M. \& Wilkins, S. W. (1972). Interatomic Potentials and Simulation of Lattice Defects, Edited by P. C. Gehlen, J. T. Beeler and R. I. J AFFEe, pp. 265-280. New York: Plenum.

Lin, W., Sprueill, J. E. \& Williams, R. O. (1970). J. Appl. Cryst. 3, 297-305.

Metcalfe, E. (1973). Ph.D. thesis, Univ. of Cambridge.

Moss, S. C. (1964). J. Appl. Phys. 35, 3547-3553.

Moss, S. C. \& Clapp, P. C. (1968). Phys. Rev. 171, 764-777.

MotT, N. F. \& Jones, H. (1936). Theory of the Properties of Metals and Alloys. New York: Dover.

Shirley, C. G. (1974). Phys. Rev. B 10, 1149-1159.

Shirley, C. G. \& Wilkins, S. W. (1972). Phys. Rev. B6, 1252-1263.

Wilkins, S. W. (1970). Phys. Rev. B2, 3935-3942.

Wilkins, S. W. (1972). Correlations and Interactions in Binary Alloys, Ph. D. thesis. Univ. of Melbourne.

Wilkins, S. W. \& ShIRLEY, C. G. (1974). To be published. Wu, D. H. \& Tahir-Kheli, R. A. (1971). J. Phys. Soc. Japan, 31, 641-648. 